\title{
Identification of Commonly Used Anthelmintic Drugs and Evaluation of their Utilization in University of Gondar Veterinary Clinic
}

\author{
Chalachew Kassahun ${ }^{1}$, Ahmed Adem ${ }^{1}$, Mebrie Zemene ${ }^{1 *}$, Gashaw Getaneh ${ }^{2}$ and Kassahun Berrie ${ }^{1}$
}

${ }^{1}$ Department of Veterinary Pharmacy, Faculty of Veterinary Medicine, University of Gondar, Gondar, Ethiopia

${ }^{2}$ Department of Biomedical Science, Faculty of Veterinary Medicine, University of Gondar, Gondar, Ethiopia

\begin{abstract}
A retrospective study was conducted from March, 2016 to June, 2016 in University of Gondar veterinary clinic to identify commonly used anthelmintic drugs and to assess the pattern of anthelmintic utilization. Data on 557 anthelmintics was collected from case registration books written for the last two years and three months (from January 01, 2014 to March 30, 2016). Out of the total anthelmintics (557), 285 (51.2\%) albendazole, $214(38.4 \%)$ ivermectin, $24(4.3 \%)$ mebendazole, $22(3.9 \%)$ fenbendazole, $7(1.3 \%)$ triclabendazole, $4(0.7 \%)$ tetramizole and 1 $(0.2 \%)$ praziquantel were utilized. $44.3 \%$ of the anthelmintics were prescribed to treat diseases that were tentatively diagnosed as nonparasitic cases. Out of the total anthelmintics utilized, $253(45.4 \%)$ were given for bovine. 227 $(40.8 \%)$ and $330(59.2 \%)$ of the anthelmintics were prescribed for young and adult animals respectively. $395(70.0 \%)$ and $167(30.0 \%)$ of the anthelmintics were also given for animals with poor body condition and good body condition, respectively. $9.5 \%, 34.6 \%, 31.8 \%$, and $24.1 \%$ of the anthelmintics were utilized in winter, spring, summer and autumn, respectively. $513(92.1 \%)$ of the anthelmintics were prescribed to treat diseases that were diagnosed empirically. The remaining $44(7.9 \%)$ were prescribed based on definitive (laboratory- supported) diagnosis. The total number of anthelmintics was prescribed by generic name. Out of the total anthelmintics, $385(69.1 \%)$ were prescribed in combination with other drugs. The findings had shown that problems of correct diagnosis, repeated use of the same class of anthelmintics for long period and prescription of anthelmintics for nonparasitic diseases. Therefore, sound veterinary diagnosis before considering the use of anthelmintics and rotation of anthelmintics between classes should be practiced.
\end{abstract}

Keywords: Anthelmintics; Retrospective study; Utilization

\section{Introduction}

In Ethiopia, animals are important sources of income for rural communities whose livelihood is largely based on livestock production [1]. However, animal production in the country is hindered by many factors including animal health constraints, inadequate nutrition and poor husbandry systems [2]. Studies in different parts of the country have shown that helminth parasites are major problems in animal production, causing mortality and production losses [3-5]. The control of parasitic helminths in domestic animals relies largely on the use of anthelmintic drugs. But inappropriate and indiscriminate use of anthelmintic leads to the emergence of anthelmintic resistance, treatment failure and increase in mortality and morbidity [6].

Most failures during anthelmintic therapy may occur when the parasite is unknown and anthelmintic drugs are administered empirically. To avoid these problems, confirmatory diagnosis and selection of the right anthelmintic should be applied [7]. Irrational use of drugs in veterinary medicine as well as the need for control of their use becomes even bigger problem when used on food producing animals. In this case, there is the possibility that minimal quantities of drugs and their metabolites (residues) which remain in edible tissues or in animal products (meat, milk, eggs, honey) induce certain harmful effects in humans as potential consumers of such food [8]. When drugs are used to improve the productivity of food animals that are intended for human consumption, then there is possibility for producing adverse effects on humans. To prevent this risk, it is necessary to use drugs rationally, i.e., to use them only when they are really indicated, in the right way, at the right time, in the right dose and respecting withdrawal period [7].

Unsound use of anthelmintics in veterinary practice, for both food producing and companion animals, favors the development of either intrinsic or acquired anthelmintic resistance. Anthelmintic drug resistance is a growing problem, and indeed developing new drugs may not be the solution for this problem. Some of the common causes that contribute to the development of anthelmintic resistance are unnecessary use of anthelmintic drugs, inappropriate dose, inadequate duration of therapy, use of irrational drug combinations [9].

Globally, more than half of all medicines are prescribed, dispensed or sold improperly, and $50 \%$ of human patients fail to take them correctly. This is more wasteful, expensive and dangerous, both to the health of the individual patient and to the population as a whole that magnifies the problem of misuse of anthelmintic agents [7]. In Ethiopia, improper utilization of anthelmintic drugs has been reported by Beyene et al. [10] in Bishoftu, Central Ethiopia. The study conducted by Melaku et al. [11] from January to September, 2011 in North Gondar also revealed that anthelmintic drugs are quite commonly but improperly utilized in the area. There is no recent published report on the utilization of commonly used anthelmintics to control helminths of animals at university of Gondar veterinary clinic in particular and in north Gondar in general.

*Corresponding author: Mebrie Zemene, Lecturer, Department of Veterinary Pharmacy, Faculty of Veterinary Medicine, University of Gondar, Gondar, Ethiopia, Tel: 251918518866; E-mail: zemenemebrie@gmail.com

Received July 12, 2016; Accepted August 31, 2016; Published September 05, 2016

Citation: Kassahun C, Adem A, Zemene M, Getaneh G, Berrie K (2016) Identification of Commonly Used Anthelmintic Drugs and Evaluation of their Utilization in University of Gondar Veterinary Clinic. J Vet Sci Technol 7: 381. doi: 10.4172/2157-7579.1000381

Copyright: (c) 2016 Kassahun C, et al. This is an open-access article distributed under the terms of the Creative Commons Attribution License, which permits unrestricted use, distribution, and reproduction in any medium, provided the original author and source are credited. 
Therefore, the objectives of the study were:

- To identify the commonly used anthelmintics in University of Gondar veterinary clinic

- To assess the pattern of anthelmintic utilization in University of Gondar veterinary clinic

\section{Materials and Methods}

\section{Study area and period}

The study was conducted from March, 2016 to June, 2016 in University of Gondar Veterinary Clinic, Gondar, North West Ethiopia, which is located at $739 \mathrm{~km}$ North West of Addis Ababa. Gondar is situated at $12.3-13.8^{\circ} \mathrm{N}$, latitude and $35.3-35.7^{\circ} \mathrm{E}$ longitude and an altitude of 2200 meter above sea level. Farmers near to Gondar town practice a mixed crop-livestock farming system [12].

\section{Study design}

A retrospective survey was carried out to identify commonly used anthelmintic drugs and to assess the pattern of anthelmintic utilization in University of Gondar veterinary clinic. The study was conducted on anthelmintic drugs utilized in University of Gondar veterinary clinic from January 01, 2014 to March 30, 2016 for the treatment of animal's patients. All anthelmintic drugs utilized from January 01, 2014 to March 30, 2016 were assessed.

\section{Data collection}

Data was collected retrospectively from case registration books in University of Gondar Veterinary Clinic. The specific data necessary to the study was recorded for each anthelmintic drug and was entered into a data record form. For this particular study, data on 557 anthelmintics that contain the treated animal's characteristics (age and body condition), disease diagnosis (name, empiric or physical clinical examination and confirmatory laboratory tests used), prescribed anthelmintics (type, naming (generic or brand), other combined drugs prescribed and route of administration were collected from case books written for the last two years and three months (from January 01, 2014 to March 3, 2016).

\section{Data analysis}

All data in the record form was entered into Microsoft Excel spread sheet and descriptive statistics (Percentage) was computed. The data was analyzed using Statistical Package for Social Sciences (SPSS) version 16 statistical software.

\section{Results}

\section{Commonly used anthelmintics}

A retrospective data was collected on a total of 557 anthelmintics utilized in University of Gondar veterinary clinic from January 1, 2014 to March 30, 2016 and analyzed. Out of the total anthelmintics (557), 285 (51.2\%) albendazole, $214(38.4 \%)$ ivermectin, $24(4.3 \%)$ mebendazole, 22 (3.9\%) fenbendazole, 7 (1.3\%) triclabendazole, 4 $(0.7 \%)$ tetramizole and $1(0.2 \%)$ and praziquantel were utilized (Table 1). $44.3 \%$ of the anthelmintics were prescribed to treat diseases that were tentatively diagnosed as nonparasitic cases (Table 2).

\section{Anthelmintic utilization based on species, age, body condition} of animals and season

Based on species of animals, out of the total anthelmintics utilized, 253 (45.4\%) were given for bovine (Table 3). 227 (40.8\%) and 330
(59.2\%) of the anthelmintics were prescribed for young and adult animals, respectively (Table 3). 395 (70.0\%) of the anthelmintics were also given for animals with poor body condition. The remaining $167(30 \%)$ were prescribed for animals with good body condition. The study indicated that the maximum anthelmintic use was observed in spring. The minimum anthelmintic use was also observed in winter (Table 3).

\section{Anthelmintic utilization by type of diagnosis and prescription}

The type of diagnosis, anthelmintic prescription and combination were also assessed. Among all anthelmintics utilized, 513 (92.1\%) were prescribed to treat diseases that were diagnosed empirically, without getting correct definitive (laboratory- supported) diagnosis. The remaining 44 (7.9\%) were prescribed based on definitive (laboratorysupported) diagnosis. The total number of anthelmintics was prescribed by generic name. Out of the total anthelmintics, 385 (69.1\%) were prescribed in combination with other drugs (multivitamin, oxytetracycline, procaine penicillin, etc.) (Table 4).

\section{Discussion}

The study revealed that anthelmintic drugs are quite commonly but improperly utilized in the clinic. Three group of anthelmintics namely benzimidazoles (Albendazole, fenbendazole, mebendazole and triclabendazole), imidazothiazole (tetramisole and levamisole) and macrocyclic lactone (Ivermectin) were used. Other alternative anthelmintics were not available. Utilization of limited group of drugs for a long period may favour the development of resistance [13]. Benzimidazoles group of anthelmintics especially albendazole was the most commonly used in the clinic. Similar findings were also reported by Melaku et al. [11] in North Gondar, Niguse et al. [14] in eastern part of Ethiopia and Kumsa et al. [15] in the Southern part of Ethiopia. This might be due to easy access of albendazole in the country and its broad spectrum nature. Benzimidazoles have been developed as broad-spectrum anthelmintic agents $[16,17]$ also reported similar scenarios in Cuba.

The percentage of anthelmintics prescribed by generic name in the present study is $100 \%$ (Table 4 ), which is in line with the standard derived to serve as ideal (100\%) [18] In the study conducted by Beyene et al. [10] in Bishoftu indicated the percentage of drugs prescribed by generic name for animal subjects was $90.1 \%$, which is lower than the current finding. A national baseline study on drug use indicators in Ethiopia in September 2002 also showed the percentage of drugs

\begin{tabular}{|c|c|c|}
\hline Anthelmintics & Frequency & Percentage (\%) \\
\hline Albendazole & 285 & 51.2 \\
\hline Ivermectin & 214 & 38.4 \\
\hline Mebendazole & 24 & 4.3 \\
\hline Fenbendazole & 22 & 3.9 \\
\hline Triclabendazole & 7 & 1.3 \\
\hline Tetramizole & 4 & 0.7 \\
\hline Praziquantel & 1 & 0.2 \\
\hline
\end{tabular}

Table 1: Percentage of anthelmintic utilized in University of Gondar veterinary clinic from January 01, 2014 to March 30, 2016.

\begin{tabular}{|c|c|c|}
\hline Characteristics & Frequency & Percentage (\%) \\
\hline Diseases treated & & \\
\hline Lungworm infection & 69 & 12.4 \\
\hline GIT parasitism & 177 & 31.8 \\
\hline Non- parasitic diseases & 247 & 44.3 \\
\hline Ectoparasite infestation & 64 & 11.5 \\
\hline
\end{tabular}

Table 2: Percentage of anthelmintic utilized based on animal diseases diagnosed in University of Gondar veterinary clinic from January 01, 2014 to March 30, 2016. 


\begin{tabular}{|c|c|c|}
\hline Characteristics & Frequency & Percentage (\%) \\
\hline Species & & \\
\hline Bovine & 253 & 45.4 \\
\hline Equine & 62 & 11.1 \\
\hline Ovine & 166 & 29.8 \\
\hline Caprine & 16 & 2.9 \\
\hline Canine & 60 & 10.8 \\
\hline Age & & \\
\hline Young & 227 & 40.8 \\
\hline Adult & 330 & 59.2 \\
\hline Body condition & & \\
\hline Good & 167 & 30.0 \\
\hline Poor & 395 & 70.0 \\
\hline Season & & \\
\hline Winter & 53 & 9.5 \\
\hline Spring & 193 & 34.6 \\
\hline Summer & 177 & 31.8 \\
\hline Autumn & 134 & 24.1 \\
\hline
\end{tabular}

Table 3: Percentage of anthelmintic utilized based on species, age, body condition and season in University of Gondar veterinary clinic from January 01, 2014 to March 30, 2016

\begin{tabular}{|c|c|c|}
\hline Characteristics & Frequency & Percentage (\%) \\
\hline Type of diagnosis & & \\
\hline Tentative & 513 & 92.1 \\
\hline Definitive & 44 & 7.9 \\
\hline Anthelmintic prescription & & 100 \\
\hline Generic name & 557 & 0 \\
\hline Trade name & 0 & \\
\hline Anthelmintic combination & & 30.9 \\
\hline Only anthelmintic & 172 & 69.1 \\
\hline
\end{tabular}

Table 4: Percentage of anthelmintic utilized based on types of diagnosis, anthelmintic prescription and combination in University of Gondar veterinary clinic from January 01, 2014 to March 30, 2016.

prescribed by generic name for human subjects was $87 \%$ [19], which is lower than the present finding $(100 \%)$. In the study conducted in 12 developing countries (human subject), the percentage of generic drugs prescribed was low in Nigeria (58\%) and Sudan (63\%) but encouraging in Tanzania (82\%) and Zimbabwe (94\%) [20,21].

Though the primary purpose of veterinary drugs is to safeguard the health and welfare of animals [22], 44.3\% anthelmintics were prescribed irrationally to treat diseases that were tentatively diagnosed as nonparasitic cases (Table 2). This may be due to unavailability of diagnostic aids for confirmatory tests, inadequate recognition of the disease and to make the treatment more broad anthelmintics can be given in combination with other drugs. Additionally, $92.1 \%$ of anthelmintics in university of Gondar veterinary clinic were utilized to treat diseases that were tentatively diagnosed without getting correct laboratory supported diagnosis (Table 4). These reveal the presence of irrational anthelmintic use. This may be due to inadequate recognition of the disease, unavailability of diagnostic aids for confirmatory tests, and absence of a right drug. The four main reasons of irrational anthelmintic prescribing are inadequate recognition of infections that lead to prescription of unnecessary drugs, inappropriate choice of route, dose and duration of anthelmintics [23].

In the present study, the percentage of anthelmintic utilization among different species of animals was highest in bovine (45.4\%), followed by ovine (29.8\%), equine (11.1\%), canine (10.8\%) and caprine $(2.9 \%)$, respectively (Table 3$)$. This may be due to the difference in feeding habits. In this study, the rate of anthelmintic utilization in adult animals (59.2\%) was higher than in young (40.5\%) (Table 4). The lower rate of anthelmintic utilization in younger animals could be most likely due to the tradition of keeping young animals homestead than letting them to travel distance in search of grass, which could be due to fears for wild predators and young animals are unable to walk long distances in search of grass. The rate of anthelmintic utilization in animals with poor body condition $(70 \%)$ was higher than in animals with good body condition (30\%) (Table 3). This variation might be associated to limited immunological response of animals with poor body condition for parasite infections. In fact the poor body condition could be due to the parasite itself or other diseases or nutritional problems. Whatever the cause, there is compromised immune response to infection in poor body condition animals that increases vulnerability to worms [24].

The study indicated the effect of season on anthelmintic utilization in different seasons. The highest utilization was observed during the spring season (Table 3). This could be ascribed to the fact that the high moisture content during these seasons that leads to increased development of larvae and abundant pasture, thus resulting in increased contact between the host and parasites. The lowest utilization rate was also found in winter (Table 3). The probable reason may be due to decreased infection of helminths due to the unfavorable environmental factors for the development and growth of most helminths species $[25,26]$. Most of the helminths species are susceptible to desiccation in dry climatic conditions that results from the high temperature at which even eggs fail to develop into infective stage [3,27,28].

In this study the high percentage of anthelmintics were prescribed in combination with other drugs (Table 4) such as oxytetracyclin, procaine and penicillin. This may be due to inadequate recognition of the disease, unavailability of diagnostic aids for confirmatory tests and prescribers' belief that better therapeutic efficacy of combined drugs.

\section{Conclusion and Recommendations}

The study revealed that anthelmintic drugs are quite commonly but improperly utilized in the clinic. There was high percentage of anthelmintic use in adult, poor body conditioned and bovine species of animals. The highest utilization was also observed during the spring season. Moreover, there was good anthelmintic prescription by generic name. However, limited group of anthelmintics were utilized. Benzimidazole group of anthelmintics especially albendazole was the most commonly used in the clinic. The finding of anthelmintic prescription showed that there were problems of correct diagnosis. Anthelmintics were prescribed irrationally to treat diseases that were tentatively diagnosed as nonparasitic cases. Additionally, anthelmintic drugs were utilized to treat diseases that were tentatively diagnosed without getting correct laboratory supported diagnosis.

Based on the above conclusions the following recommendations are forwarded:

- Adequate diagnostic aids for confirmatory diagnosis should be available/full-filled in the clinic.

- Sound veterinary diagnosis must be carried out before considering the use of anthelmintics.

- Awareness should be created on the use of anthelmintics to avoid using anthelmintics for nonparasitic diseases.

Frequent and repeated use of anthelmintics from the same class, over an extended period of time may favour the development of resistance so rotation of anthelmintics between classes should be practiced. 
Citation: Kassahun C, Adem A, Zemene M, Getaneh G, Berrie K (2016) Identification of Commonly Used Anthelmintic Drugs and Evaluation of their Utilization in University of Gondar Veterinary Clinic. J Vet Sci Technol 7: 381. doi: 10.4172/2157-7579.1000381

\section{References}

1. Metaferia F, Cherenet T, Gelan A, Abnet F, Tesfay A, et al. (2011) A Review to Improve Estimation of Livestock Contribution to the National GDP. Ministry of Finance and Economic Development and Ministry of Agriculture. Addis Ababa, Ethiopia.

2. Tembely S, Lahlou-Kassi K, Rege JE (1998) Breed and season effects on the peri-parturient rise in nematode egg output in indigenous ewes in a cool tropical environment. Vet Parasitol 77: 123-132.

3. Negassa A, Rashid S, Gebremedhin B (2011) Livestock production and marketing in Ethiopia. Ethiopian Support Strategy Program II (ESSP II) Working Paper 26. International Food Policy Research Institute, Washington, D.C., USA.

4. Abebe W, Esayas G (2001) Survey of ovine and caprine gastro-intestina helminthosis in eastern part of Ethiopia during the dry season of the year. Rev Med Vet 152: 379-384.

5. Biffa D, Jobre $Y$, Chakka $\mathrm{H}$ (2006) Ovine helminthosis, a major health constrain to productivity of sheep in Ethiopia. Anim Health Res Rev 7: 107-118.

6. Taylor MA, Coop RL, Wall RL (2007) Veterinary Parasitology. 3rd edn Blackwell Publisher, Ltd., UK, pp: 195-198.

7. Ćupić V, Dobrić S, Antonijević B, Čelebićanin S (2011) The significance of rational use of drugs in veterinary medicine for food safety. Tehnologija mesa 52: 74-79.

8. Sanders $P$ (2007) Veterinary drug residue control in the European Union Technologija mesa 1: 59-68.

9. AAVP (2005) Anthelmintic Resistance: An Examination of its Growing Prevalence in the US cattle herd. Executive Summary of the 2005 Anthelmintic Resistance Roundtable. pp: 1-8

10. Beyene T, Endalew D, Tolossa Y, Feyisa, A (2015) Evalution of rational use of veterinary drugs especially antimicrobials and anthelmintics in Bishoftu, Central Ethiopia. BMC Res Notes 8: 482.

11. Melaku A, Bogale B, Chanie M, Fentahun T, Berhanu A (2013) Study on the utilization and efficacy of commonly used anthelmintics against gastrointestinal nematodes in naturally infected sheep in North Gondar, North-Western Ethiopia. Academic Journals 7: 679-684.

12. CSA (2007) Census Central Statistics authority. Ethiopian agricultural enumerations. Result for Amhara region.

13. Papadopoulos E (2008) Anthelmintic resistance in sheep nematodes. Smal Rumin Res 76: 99-103.

14. Niguse A, Shimelis D, Feyera T (2014) Epidemiology and Chemotherapy of Gastrointestinal Parasites of Sheep in and Around Jigijga, Eastern Ethiopia. Eur J Biol Sci 6: 46-53.
15. Kumsa B, Debela E, Megersa B (2010) Comparative efficacy of Albendazole, Tetramisole and Ivermictin against gastrointestinal nematodes in naturally infected goat in Ziway Oromia Regional state (South Ethiopia). J Anim Vet Adv 9: 2905-2911.

16. Brunton L, Goodman LS (2008) Goodman and Gilman's Manual of Pharmacology and Therapeutics. McGraw-Hill Professional, Pp: 697-709.

17. Arece J, Mahieu M, Archimède H, Aumont G, Fernández M, et al. (2004) Comparative efficacy of six anthelmintics for the control of gastrointestinal nematodes in sheep in Matanzas, Cuba. Small Rumin Res 54: 61-67.

18. Isah AO, Ross-Degnan D, Quick J, Laing R, Mabadeje AFB (2004) The development of standard values for the WHO drug use prescribing indicators. International conference on improving use of medicines (ICIUM). INRUDNigeria1, Support Group 2; DAP-WHO3. Nigeria.

19. EPA (2003) Assessment of the Pharmaceutical Sector in Ethiopia. Addis Ababa, Ethiopia.

20. Bimo D (1993) Report on Nigerian field test. INRUD News. 1992; 3: 9-10. How to investigate drug use in health facilities. Geneva: WHO, p: 74

21. WHO (2002) Promoting rational use of medicines: core components. WHO Policy and perspectives on medicine. Geneva.

22. Cannavan A (2004) Capacity Building for Veterinary Drug Residue Monitoring Programmes in Developing Countries. Joint FAO/WHO Technical Workshop on Residues of Veterinary Drugs without ADI/MRL - Bangkok, 24-26 August 2004.

23. Pallares R, Dick R, Wenzel RP, Adams JR, Nettleman MD (1993) Trends in antimicrobial utilization at a tertiary teaching hospital during a 15 year period (1978-1992). Infect Control Hosp Epidemiol 14: 376-382.

24. Sykes AR (2010) Host immune responses to nematodes: benefit or cost? Implications for future development of sustainable methods of control. R Bras Zootec 39: 376-382.

25. Andrews SJ (1999) The life cycle of Fasciola hepatica. In: Fasciolosis. Dalton JP (ed). CAB, Wallingford, pp: 1-29.

26. Lima WS (1998) Seasonal infection pattern of gastrointestinal nematodes of beef cattle in Minas Gerais State-Brazil. Vet Parasitol 74: 203-214.

27. Banks DJD, Singh R, Barger IA, Pratap B, Le Jambre LF (1990) Development and survival of infected larvae of Haemonchus contortus and Trichostrongylus colubriformis on pastures in a tropical environment. Int J Parasitol 20: 155-160.

28. Waruiru RM, Mutune MN, Otieno RO (2005) Gastrointestinal parasite infections of sheep and goats in a semi-arid area of Machakos District, Kenya. Bull Anim Health Prod Afr 53: 25-34. 